INNOVATIONS IN PRACTICE

\title{
The Calgary COPD \& Asthma Program: The role of the respiratory therapy profession in primary care
}

\author{
Cindy L. Slack, RRT, CRE, CTE ${ }^{1}$, Kathy Hayward, BSP, CRE, CTE ${ }^{1}$ Andrea White, Markham, RRT, CRE, CTE
}

CL Slack, K Hayward, AW Markham. The Calgary COPD \& Asthma Program: The role of the respiratory therapy profession in primary care. Can J Respir Ther 2018;54(4):86-91. doi: 10.29390/cjrt-2018-018.

\begin{abstract}
Respiratory therapy is a profession with the depth and breadth required to care for clients by "evaluating, treating, and maintaining cardiopulmonary (heart and lung) function" [1]. Registered Respiratory Therapists (RRTs) work in a variety of settings from critical and acute care to primary and home care. After a brief overview of the models and competencies that underpin the work of the Calgary COPD \& Asthma Program, the article provides a description of the program and discuss how RRT Certified Respiratory Educators provide services within the program. These services include health promotion through self-management education rooted in the Chronic Care Model [2] and supported by the National Alliance of Respiratory Therapy Regulatory Bodies' National Competency Framework for the Profession of Respiratory Therapy [3]. The authors hope the success of this program will encourage others to embrace the role of the RRT in a primary health care setting.
\end{abstract}

Key Words: respiratory education; primary health care; RRT; certified respiratory educator (CRE); community sites/services

\section{BACKGROUND}

Support for the role of respiratory therapy in primary health care is grounded in literature regarding the management of chronic disease and the profession's scope of practice. It is therefore helpful to examine these, and other foundational constructs, to provide context for the discussion.

\section{Chronic Care Model}

Two decades ago, Wagner's Chronic Care Model called for a transformation of health care from the medical model (diagnose and treat acute conditions) to one that was focused on long-term management of chronic or incurable conditions [4]. Wagner identified key elements that work together to improve the outcomes of those living with chronic disease [5]:

(1) delivery system design from single care giver to teams of clinical practitioners with defined roles collaborating to support management of chronic disease;

(2) decision support through the use of evidence and guidelines and patient preferences;

(3) self-management support through collaboration, goal setting, and refining therapy to enable individuals to manage their chronic disease(s);

(4) community resources that are known and utilized and support individuals outside the traditional health system;

(5) a health system that promotes safety and pursues excellence, including communication of errors and improvements;

(6) clinical information systems where computerized disease management information is readily available and shared with the team and patient.
The model projects that these elements will result in a collaboration between "informed, activated clients" with "prepared proactive practice teams" and improved outcomes with decreased cost $[2,4]$.

\section{Respiratory therapy scope of practice}

Registered Respiratory Therapists (RRTs) bring a unique skill set to individuals who require respiratory care from the acute care setting to primary health care. In Canada, the National Alliance of Respiratory Therapy Regulatory Bodies (NARTRB) provides direction on the scope of practice for the profession of respiratory therapy [3]. The NARTRB's National Competency Framework (NCF) for the Profession of Respiratory Therapy has nine core competencies (and their 43 elements) and 10 clinical competencies (and their 36 elements) that provide this information [3]. Although there are some critical care and anaesthesia elements that may not be practiced in primary health care, others are routinely practiced and some appear to be specifically directed to these settings, for example:

- B4 Optimize cardio-respiratory health and wellness of the community (core competency) [3]

- B4.1 Provide cardio-respiratory health education (element)

- B4.2 Participate in addressing cardio-respiratory health needs of the community (element)

Other competencies indicate higher expertise for RRTs, including those working in primary health care [3], for example:

- B0.2.4 Participate in improving respiratory care policies and protocols

- B0.3.6 Develop respiratory treatment protocols/C1.3.2 Develop respiratory treatment protocols

${ }^{1}$ Calgary COPD $\mathcal{E}$ Asthma Program, Alberta Health Services, Calgary, AB, Canada

${ }^{2}$ Canadian Network for Respiratory Care, Eldorado, ON, Canada

Correspondence: Cindy L. Slack, RRT, CRE, CTE, Calgary COPD Ë Asthma Program, Alberta Health Services, NWI 4500-16 Avenue NW, Calgary, AB T3B 0M6, Canada. Tel: 403-809-3715, Fax: 403-283-3406; E-mail: cindy.slack@ahs.ca 
- B4 Optimize cardio-respiratory health and wellness of the community

- ...supplemental certifications include: certified asthma educator...certified respiratory educator and certified tobacco educator.

-B8.1.5/B8.2.5 Drive progress and evolution of professional practice

- C2.2.6 Identify process or system gaps involving risks to patient safety

Primary health care and medical home defined

Primary care and primary health care are at times used interchangeably. This paper will use the terminology "primary health care" based on the discussion by Health Canada: "...an approach to health and a spectrum of services" including "direct provision of first contact services..." and "coordination function to ensure continuity and ease of movement across the system so that care remains integrated..." [6]. Primary health care in the context of this paper includes family physician's offices, certified respiratory educator led community clinics, home care, community pharmacies, and chronic disease education and exercise programs. The flexibility of RRTs providing home care has been recognized to bring positive results in both client outcomes and resource utilization [7].

Medical home is "a model of primary care that is patient-centered, comprehensive, team-based, coordinated, accessible, and focused on quality and safety" [8]. In Canada, the College of Family Physicians of Canada's vision of the "patient's medical home" is complex, speaking of where the patient feels most "at home" to discuss health concerns; where patients are active participants in decision-making, and care givers collaborate with each other and the patient to provide a comprehensive range of services; and where the therapeutic relationships result in "the best possible health outcomes for each person, the practice population, and the community being served" [9].

\section{INTRODUCTION}

In its first iteration, the Calgary COPD \& Asthma Program (CCAP) worked with adults with asthma. Over time it developed into its current format as a comprehensive multidisciplinary program for adults with asthma, chronic obstructive pulmonary disease (COPD), chronic cough, and tobacco use disorder.

Asthma is a chronic lung disease requiring ongoing medical care that affects approximately 2.74 million Canadians and causes the death of almost 250 Canadians annually [10]. The economic burden of asthma is caused by the high costs of uncontrolled asthma due to increased health care utilization and lost productivity as well as the medical visits and medication costs for those whose asthma is in control [11]. The National Asthma Education Program (NAEP) in 1991 endorsed four major components to asthma care [12]: (i) client education, (ii) environmental control to avoid acute exacerbations, (iii) pharmacologic therapy, and (iv) the use of objective measurement to assess severity and monitor asthma therapy.

To achieve these recommendations, a multidisciplinary team approach is required to provide access to prescribed medication and client education by qualified educators to support and reinforce self-management strategies. As there was no such coordinated approach to the management of asthma in Alberta, the CCAP (called the Calgary Asthma Program at that time) was established at Foothills Medical Hospital to address the needs of adults with asthma in the Calgary area. The goal of the program was to decrease asthma morbidity and mortality.

COPD is a progressive, disabling, chronic lung disease and is currently the fourth most common cause of death in Canada. COPD accounts for approximately $4.6 \%$ of all deaths [13] and is the leading cause of hospitalizations [14]. The World Health Organization (WHO) projects COPD to be the third largest cause of death worldwide by 2030. In Canada, the prediction is for a $155 \%$ increase in the diagnosis of COPD and $210 \%$ increase in hospitalizations in the same timeframe [14].
Tobacco use disorder is listed in the Diagnostic and Statistical Manual of Mental Disorders as a "maladaptive pattern of nicotine use, leading to significant impairment of distress," for example, tolerance, withdrawal symptoms, repeated unsuccessful quit attempts, desire to quit etc. [15]. The smoking rate in Alberta is $15.8 \%$ compared with Canada's smoking rate of $13 \%$ (excluding territories) [16]. Tobacco smoking is a major risk factor in developing COPD and smoking cessation is the single most effective intervention to reduce the risk of developing COPD. It is the only intervention that has been shown to slow the rate of lung function decline [17]. Exposure to tobacco smoke, either by active or passive smoking, is a major factor in triggering asthma by increasing symptoms, increasing loss of lung function, and decreasing efficacy of inhaled and oral corticorsteroids $[18,19]$.

Chronic cough accounts for $38 \%$ of outpatient referrals to respiratory specialists and although some cases are caused by life-threatening disease, (e.g., lung cancer and heart disease), others are chronic conditions that require less invasive management strategies including chronic lung diseases, gastroesophageal reflux, laryngeal irritation, etc. [20].

The Calgary Asthma Program began with one staff asthma educator who provided client education and a respirologist who provided medical direction and prescriptions when required. Because the client needs included other health concerns and there was increasing demand for service, the program expanded to include other respiratory health concerns. COPD, tobacco use disorder, and chronic cough and were added to the program, and it was renamed to CCAP.

This paper will explore the CCAP alignment with the Expanded Chronic Care Model and allows the RRT Certified Respiratory Educators (CREs) working in the program to maximize the NCF core competencies and to develop as leaders in clinical skills related to chronic disease management skills (See National Competency Framework for the Profession of Respiratory Therapy) [3].

\section{DISCUSSION OF THE APPLICATION OF THE CHRONIC CARE MODEL}

\section{Organization of health care: Delivery system design}

CCAP is a comprehensive multidisciplinary program for adults with asthma, COPD, chronic cough, and tobacco use disorder. It is an outpatient service that takes a preventative health approach to help individuals gain control of and improve their health [21]. Using objective measures (spirometry) and subjective assessment the CCAP CREs help confirm diagnosis and disease severity. Through collaboration with prescribers, pharmacological and nonpharmacological optimization is made for each client. Support for individuals and families living with respiratory disease is provided through client education that is focused on self-management.

The goal of the program is to improve clients' quality of life. The mission statement of CCAP is "To improve the lives of those affected by asthma, COPD, tobacco, and chronic cough by providing lung health care and leadership through education and self-management skills" [21].

The move from an acute or physician-centered model of care to a community-based model emphasizing individualized client-centered care with education as a key component was discussed at conferences for asthma educators. In response, the program expanded into primary care in 1998. Because of referral patterns, requests from clinicians, and client characteristics, the program expanded its staffing and also began to accept referrals for clients with COPD or tobacco use disorder. It also supported the relationship between client and primary care giver by providing specialized respiratory care in the client's medical home. Funding stabilized in 2002 when the Calgary Health Region began to cover the costs of CCAP CREs working with respirologists in specialty outpatient clinics. In 2005 the program was incorporated into what is now known as Alberta Health Services (AHS) giving the program added stability.

\section{Decision support}

A cross-sectional review of asthma control and treatment in Alberta communities demonstrated that there had been no significant improvement 
in asthma control or use of action plans between 1997 and 2002, leading the respirologists and CCAP CREs authors to conclude that "there was no evidence that patient education had increased" [22]. A move was then made to expand further into the community with the development of CRE-run community clinics in 2006.

Referrals are accepted from acute care hospitals, emergency departments, and primary care providers and clients can self-refer. The information provided to clients is based on current best practices and guidelines, and it is delivered using proven education methods by CCAP CREs. To support the client, the CCAP CRE may contact the primary care provider by telephone while the client is present about suggested changes to the treatment plan based on current guidelines. CCAP CREs are also able to access a respirologist in a timely fashion to facilitate prompt intervention when necessary. A report is sent back to the primary care physician after each visit and includes findings, recommendations based on guidelines, and results of objectives measures of spirometry with interpretation.

Follow-up can be provided through CCAP CREs, primary care providers, and community pharmacists.

\section{Organization of health care: self-management support}

Clients meet their educator for a one-on-one session that is individualized based on the client's needs, goals, and characteristics. Sessions can include a variety of interventions, for example assessment of disease and quality of life, goal setting, education on self-management, and behaviour change counselling. Goal setting is collaborative and provides the CCAP CRE with a client-centred approach. Central to the client education is the development of an individualized written action plan based on the current guidelines for their disease. Its development occurs through a collaboration with the client, educator, and prescriber; the client provides insight into what will work for them and how the plan can be adjusted to meet their specific needs, for example trigger management, negotiations on medication, or specific inhaler use. The plan is based on self-management during times of relative wellness and worsening or exacerbation using current national and international guidelines. Plans include information related to ongoing day-to-day management (wellness - green zone); recognition of a flare up of symptoms, direction on actions to take including how much medication, for how long and what symptoms warrant escalation to medical attention (worsening/exacerbation - yellow zone); and when and how to seek medical attention (more severe worsening/exacerbation - red zone) [18, 23-25]. Education on self-management includes areas related to skill acquisition, e.g., optimizing inhaler technique and symptom based self-regulation (self-observation of symptoms/interpretation of meaning of symptoms/reaction to symptoms) and methods of enhancing the client's confidence in changing their medication [18, 23-25]. Counseling on behaviour change includes adherence to the plan, trigger management and avoidance, and tobacco (prevention, protection, and cessation), and it is delivered through the application of models and theories, e.g., Social Cognitive Theory [26], Health Belief Model [27], Transtheoretical Model [28].

For clients who require more support, the educator offers to link the client to community and other health resources through referrals, for example: Alberta Healthy Living Program [29] for exercise, nutritional counseling and general health education; pulmonary rehabilitation through Community Accessible Rehabilitation that is offered at two AHS sites; and Alberta Quits [30], a province wide service offering smoking cessation help through online self-help and/or phone, text or group counseling.

\section{RESULTS}

\section{Prepared, proactive practice team}

CCAP CRE staffing is currently eight health care professionals. Originally mainly nursing staff, staffing mixes have included respiratory therapists, physiotherapists, and pharmacists; currently staffing is seven RRTs and one pharmacist. CCAP CREs have brought their unique skills and in-depth knowledge regarding the diagnosis and management of chronic respiratory diseases, e.g., their expertise in the use of spirometry and other objective measures of lung health, competence in pharmacotherapy including inhaled medication delivery devices, and interprofessional collaboration [3]. A CCAP CRE works primarily as the sole educator in a given setting. To ensure standardized education and counseling is provided across the program and to continue to build the body of knowledge of the team, the CCAP CREs meet regularly to review literature, update their protocols, and share experiences, e.g., difficult to manage cases with spirometries are done following current guidelines and provincial standards.

The RRTs in CCAP have obtained post-graduate education and certification as a certified respiratory educator, and many have recently also gained their certified tobacco educator. This builds on client education, chronic respiratory disease management, and tobacco cessation training and counseling that are part of the RRT clinical competencies [3]. It includes health promotion and human development as it applies to health education, education theories, instructional design, and counseling methods including motivational interviewing and program evaluation [31]. Certification as a respiratory educator or tobacco educator will allow a RRT to develop the skills and knowledge in the management of chronic respiratory disease as a step to expand their scope of practice [3].

A study of the differences in asthma management in primary care between rural and urban centres found that although care was comparable, both settings required improvements in use of spirometry, delivery of asthma education, and use of written action plans [32]. The addition of RRTs facilitated the use of spirometry in primary care, assisted primary care practitioners in confirming asthma, determined the efficacy of asthma treatment, diagnosed or excluded COPD, and staged clients with COPD $[18,23-25]$. Use of spirometry to improve asthma and COPD management has increased awareness of its importance, and resulted in direct referrals from other members of the multidisciplinary team to CCAP.

The NCF identifies expertize through recognition of gaps in care and development of services to meet the gap [3]. This is exemplified in CCAP by the CRE-led Chronic Cough Clinic, established in 2006. The clinic's function is to provide prompt and cost-effective care to clients with chronic cough not related to other respiratory disorders or managed by traditional therapy [20]. The Chronic Cough Clinic has shown an improvement in cough resolution and client quality of life since its inception and shown that clients managed by CREs have similar outcomes to those managed by respirologist. Another effect was the decanting clients from the wait lists of the local specialists, reducing wait times for respirology consultation from 2 months to less than 4 weeks [20]. Clients who are referred to the clinic are assessed for inclusion/exclusion criteria (see Table 1). The CCAP CRE and respirologist collaborate

\section{TABLE 1}

CCAP Chronic Cough Clinic inclusion and exclusion criteria [20]

\begin{tabular}{|c|c|}
\hline Inclusion criteria & Exclusion criteria \\
\hline Age - 18 years or older. & $\begin{array}{l}\text { Systemic symptoms - weight loss, night } \\
\text { sweats, fatigue/malaise. }\end{array}$ \\
\hline $\begin{array}{l}\text { Cough - unexplained and } \\
\text { greater than } 4 \text { weeks duration. }\end{array}$ & $\begin{array}{l}\text { Respiratory disease - active } \\
\text { tuberculosis, bronchiectasis, COPD, } \\
\text { asthma. }\end{array}$ \\
\hline Spirometry - normal. & $\begin{array}{l}\text { Cancer - history of cancer (non- } \\
\text { melanoma skin cancer acceptable). }\end{array}$ \\
\hline $\begin{array}{l}\text { Chest x-ray - normal or minor } \\
\text { abnormalities unlikely to explain } \\
\text { cough, e.g., calcified granuloma. }\end{array}$ & $\begin{array}{l}\text { Cardiac disease - myocardial infarction } \\
\text { within past } 12 \text { months, heart failure within } \\
\text { last } 12 \text { months. } \\
\text { Blood disorders - examples: anemia, } \\
\text { platelet disorders, coagulation disorders, } \\
\text { neutropenia, lymphocytopenia. } \\
\text { Other systemic diseases, examples: } \\
\text { collagen disorders (temporal arteritis, } \\
\text { Ehlers-Danlos syndrome, systemic lupus } \\
\text { erythematous), Chron's disease or } \\
\text { dysphagia. }\end{array}$ \\
\hline
\end{tabular}


The Calgary COPD \& Asthma Program

TABLE 2

Education provided by the CCAP Educators

\begin{tabular}{|c|c|c|}
\hline Target audience & Topics & Discussion \\
\hline $\begin{array}{l}\text { University of Calgary first-year medical } \\
\text { students }\end{array}$ & $\begin{array}{l}\text { Motivational interviewing for smoking cessation } \\
\text { and prevention; Transtheoretical Model of Staged } \\
\text { Change; ncotine replacement therapy; Alberta Quits }\end{array}$ & $\begin{array}{l}\text { Developed by CCAP in 1996; acilitated by CCAP } \\
\text { educators and local CREs on an annual basis. }\end{array}$ \\
\hline Health professionals & $\begin{array}{l}\text { CCAP process and structure, e.g., how to identify, } \\
\text { manage and refer clients with asthma, COPD, etc. }\end{array}$ & Biannual training. \\
\hline SAIT respiratory therapy students & Mentorship & $\begin{array}{l}\text { By request the student shadows an educator in } \\
\text { primary health care setting for up to } 1 \text { full day. }\end{array}$ \\
\hline University of Alberta pharmacy students & Mentorship & $\begin{array}{l}\text { By request the student shadows an educator in } \\
\text { primary health care setting for up to } 1 \text { full day. }\end{array}$ \\
\hline $\begin{array}{l}\text { Health care providers and general public, } \\
\text { www.ucalgary.ca/asthma }\end{array}$ & $\begin{array}{l}\text { Medically accurate information on the management } \\
\text { of chronic respiratory diseases. }\end{array}$ & $\begin{array}{l}\text { Includes all inhaled medication devices that are used } \\
\text { across the province; information provided at a level } \\
\text { appropriate for general public. }\end{array}$ \\
\hline
\end{tabular}

to determine a diagnosis and a treatment plan for each client and then jointly present the plan to the client. The CCAP CRE and client work together to build the client's skills and confidence to self-manage their chronic cough. Follow-up occurs as needed by telephone. Further investigation, such as computed tomography of the chest, induced sputum analysis or methacholine challenge testing, is arranged when required.

A randomized controlled trial examining the efficacy of CCAP CRE led chronic cough clinics compared with those led by a respirologist found that CCAP CREs provide more contacts per client (4.9 vs. 2.7; $p$ $\leq 0.0001)$ and have more clients with resolved cough $(p \leq 0.02)$. Although both groups had significant improvements in quality of life, no statically significant differences in were found between groups [20].

Another area where CCAP CREs demonstrate their expertise is their leadership in the delivery of health education to other professionals on topics related to chronic respiratory disease management and education techniques. Some of the target audiences and topics can be found in Table 2.

The CCAP is positioned to provide targeted education on selfmanagement that includes personalized written action plans for clients with diverse respiratory and related conditions. These clients then return to their medical home with new skills in health literacy (the ability to control and improve their health) [33] including management of their disease in part through self-regulation e.g. self-observation, self-evaluation and self-reaction, in part using a written action plan. WHO defines health literacy as "the ability of individuals to gain access to, understand, and use information in ways which promote and maintain good health" [34]. In a recent Cochrane Review, Howcroft found generally reliable evidence that the provision of COPD action plans with supporting education improves self-management behaviour, e.g., self-starting treatment and reduced hospital visits [34]. A 2017 systematic mega-review confirmed improved asthma control and resultant reduction in acute health care utilization through supportive self-management strategies in diverse groups without significant increase in health care costs [35].

CCAP CREs brought their expertise to a variety of settings: primary care (physician offices and CRE led clinics), acute care (inpatient bedside visits), and specialist care (respirology offices), providing education and counseling to 3297 clients in 2017-2018. As CREs, RRTs are able to work with clients with a wide variety of respiratory and related chronic diseases and CCAP now services a diverse client population, with asthma and COPD continuing to be the largest group (see Table 3 for main diagnosis of CCAP clients).

Unfortunately, funding for outcome monitoring is not available since the switch from paper to computer charting in 2009. Support for the services offered by CCAP can be found in current literature. A multisite study of outcomes of standardized asthma education demonstrated substantial improvements in the burden of asthma for those seen by the CCAP CREs (unscheduled physician visits, emergency visits, hospital utilization, missed work, and school or leisure days as well as significant improvements in quality of life) [37]. Numerous reviews of client
TABLE 3

Calgary COPD \& Asthma Program Utilization [36]

\begin{tabular}{lr}
\hline \multicolumn{2}{c}{ Main diagnosis (1 April 2017-31 March 2018) } \\
\hline Asthma, unspecified without stated status asthmaticus & $41 \%$ \\
Chronic obstructive pulmonary disease, unspecified. & $32 \%$ \\
Cough & $15.5 \%$ \\
Other specified respiratory disorders & $7.5 \%$ \\
Dyspnoea & $1.5 \%$ \\
Tobacco use disorder & $1.5 \%$ \\
Other sleep disorders & $<1 \%$ \\
Other diseases of vocal cords & $<1 \%$ \\
Chronic rhinitis & $<1 \%$ \\
Wheezing & $<1 \%$ \\
Chronic sinusitis, unspecified & $<1 \%$ \\
\hline
\end{tabular}

education aimed at self-management of asthma and COPD also support the services offered by CCAP to reduce acute health care utilization, COPD, and asthma exacerbation and to support improved lung function $[34,35,38-45]$.

\section{CONCLUSIONS}

Mable and Marriot's [46] updated description of primary health care recognizes the broader determinants of health and includes coordinating, integrating, and expanding systems and services to provide more population health, sickness prevention, and health promotion, not necessarily just by physicians. It encourages the best use of all health providers to maximize the potential of all health resources [46]. In Calgary, the CCAP's role in primary care is to provide health promotion activities that prevent the burden of illness for individuals living with chronic lung disease. By working within a chronic disease management framework promoting wellness and health literacy, CRE RRTs at CCAP have advanced their practice as experts in chronic respiratory disease management including the provision of cardio-respiratory education. Their expertise is evident through direct interventions that optimize the respiratory wellness of their clients; collaboration with, and education of, other members of the health care team to improve the respiratory wellness in Calgary; and their recognition of service gaps and development of protocols to meet these gaps. They encourage RRTs to consider working in primary care and to further develop their expertise in chronic respiratory disease and then how to apply these newly acquired skills to those in need of their specialized care.

We leave the last words on the importance of CREs from a referring family physician and the Medical Director of CCAP:

I have been fortunate to have had a CRE in my practise for almost 25 years. As a family physician it is always a joy to work with allied health care professionals since they assist me so much in the care of my patients and allow me to learn more about what they can offer and assist me in the care of patients. With the CREs in particular I have found the time they spend 
with my patients in motivational counselling, reviewing device technique, education, and spirometry has been invaluable in terms of obtaining correct diagnoses, ensuring better adherence to treatment and motivating patients to care for their respiratory health. I would be truly lost without them.

\section{Margaret Churcher BSc (Hons), MD CCFP FCFP Mosaic Primary Care Network, Calgary, Alberta}

Certified Respiratory Educators play an essential role in improving the quality of life of clients with lung disease. They also play a key role in decreasing readmission rates to hospital after flares of both asthma and COPD through teaching clients how to use their preventative medications, how to improve their exercise capacity to promote healthy living and how to recognize deterioration of their health early so that treatment can be started before another hospital admission becomes necessary. Without CREs in the community our clients have fewer options in terms of access to timely advice and interventions and the result is more frequent and longer hospital stays.

\section{Brandie Walker MD PhD FRCPC} Respirologist

Division of Respiratory Medicine, University of Calgary Medical Director, Calgary COPD E Asthma Program

\section{REFERENCES}

1. Canadian Society of Respiratory Therapists. Respiratory therapist: What is a respiratory therapist? [Internet]. The Canadian Society of Respiratory Therapists. 2018. Available at: http://www.csrt.com/respiratory-therapist (accessed May 31, 2018).

2. Wagner EH. Chronic disease management: What will it take to improve care for chronic illness? Eff Clin Pract 1998;1(1):2-4.

3. The National Alliance of Respiratory Therapy Regulatory Bodies. National competency framework for the profession of respiratory therapy, 2016 NCF Part 1 [Internet]. Ottawa; 2016. Available at: http:// www.csrt.com/2016-national-competency-framework/ (accessed May 31, 2018).

4. The MacColl Center for Health Care Innovation. The chronic care model. Improving chronic illness care, 2006-2018. Available at: http:// www.improvingchroniccare.org/index.php? $\mathrm{p}=$ The_Chronic_Care_ Model\&s=2 (accessed October 08, 2018).

5. The MacColl Center for Health Care Innovation Model Elements. The chronic care model. Improving chronic illness care, 2006-2018. Available at: http://improvingchroniccare.org/index.php?p=Model_ Elements\&s=18 (accessed October 08, 2018).

6. Health Canada. About primary health care. Health Services. Government of Canada, 2012. Available at: https://www.canada.ca/en/health-can$\mathrm{ada} /$ services/primary-health-care/about-primary-health-care.html\#a2 (accessed September 12, 2018).

7. Perrigrine L. Respiratory therapists in home care. Caring 2003; 22(1):16-19.

8. Patient-Centered Primary Care Collaborative. Defining the medical home. Pcpcc.org. 2018. Available at: https://www.pcpcc.org/about/ medical-home (accessed May 31, 2018).

9. The College of Physicians and Surgeons of Canada. A vision for Canada: Family practice - The patient's medical home. Resources. [Online] 2018. https://www.cfpc.ca/uploadedFiles/Resources/Resource_Items/ PMH_A_Vision_for_Canada.pdf (accessed September 12, 2018).

10. Stats Canada. Deaths and mortality rate, by selected grouped causes, age group and sex, Canada: Annual. Table 102-0551 Www5.statcan. gc.ca. 2018. Available at: http://www5.statcan.gc.ca/cansim/a26?lang=eng\&id=1020551 (accessed May 31, 2018).

11. Ismaila A, Sayani A, Marin M, Su Z. Clinical, economic, and humanistic burden of asthma in Canada: A systematic review. BMC Pulm Med [Internet]. 2013;13(1). Available at: https://www.ncbi.nlm.nih.gov/ pmc/articles/PMC4235031/ (accessed May 31, 2018).

12. National Asthma Education Program: Guidelines for the Diagnosis and Management of Asthma. Bethesda, MD: National Heart, Lung, and Blood Institute; 1991. US Dept of Health and Human Services Publication NIH 91-3042[Internet]. Available at: https://www. jacionline.org/article/0091-6749(91)90002-6/pdf (accessed September 12, 2018).
13. Statistics Canada. Leading causes of death, total population, by age group. Statcan.gc.ca. 2018. Available at: http://www.statcan.gc.ca/tables-tableaux/sum-som/101/cst01/hlth36a-eng.htm (accessed May 31, 2018).

14. Khakban A, Sin D, FitzGerald J, et al. The projected epidemic of COPD hospitalizations over the next 15 years: A population based perspective. Am J Respir Crit Care Med 2016;195(3):287-91. doi: 10.1164/ rccm.201606-1162PP.

15. American Psychiatric Association. Diagnostic and statistical manual of mental disorders, 5th edition. Washington, DC: APA; 2013.

16. Health Canada. Canadian Tobacco Alcohol and Drugs (CTADS): 2015 summary [Internet]. 2017. Available at: https://www.canada.ca/en/ health-canada/services/canadian-tobacco-alcohol-drugs-survey/2015. summary.html (accessed June 24, 2018).

17. O'Donnell DE, Aaron S, Bourbeau J, et al. Canadian Thoracic Society recommendations for the management of chronic obstructive pulmonary disease - 2007 update. Can Respir J 2007;14: (Suppl B):5B-32B. Available at: https://www.ncbi.nlm.nih.gov/pmc/articles/ PMC2806792/ (accessed June 24, 2018).

18. 2018 GINA Report: Global strategy for asthma management and prevention. Global Initiative for Asthma - GINA. [Internet] 2018. Available at: http://ginasthma.org/2018-gina-report-global-strategy-for-asthma-management-and-prevention/ (accessed June 3, 2018).

19. Tamimi A, Serdarevic D, Hanania NA. The effects of cigarette smoke on airway inflammation in asthma and COPD: Therapeutic implications. Respir Med 2012;103(3):319-28. doi: 10.1016/j.rmed.2011.11.003.

20. Field S, Conley D, Thawer A, Leigh R, Cowie R. Assessment and management of patients with chronic cough by certified respiratory educators: A randomized controlled trial. Can Respir J [Internet]. 2009;16(2):49-54. Available at: https://www.ncbi.nlm.nih.gov/pmc/ articles/PMC2687561/ (accessed May 31, 2018).

21. Calgary COPD \& Asthma Program. Calgary COPD \& Asthma Program: Welcome to the Calgary COPD \& Asthma Program website!. Alberta Health Services [Internet]. 2018. Available at: http://www.ucalgary.ca/ asthma/ (accessed May 31, 2018).

22. Cowie RL, Underwood MF, Sin DD, et al. Asthma control and management in the community: Indices in 1997 compared with indices in 2002. Can Fam Physician [Internet]. 2006;52(6):750-3. Available at: https://www.ncbi.nlm.nih.gov/pmc/articles/PMC1780155/ (accessed September 14, 2018).

23. Lougheed M, Lemiere C, Ducharme F, et al. Canadian Thoracic Society 2012 Guideline Update: Diagnosis and management of asthma in preschoolers, children and adults: Executive summary. Can Respir J [Internet] 2012;19(6):e81-8 Available at: https://cts-sct.ca/ wp-content/uploads/2018/01/ASTHMA-GUIDELINE-APRIL-2012. pdf (accessed May 31, 2018).

24. Bourbeau J, Bhutani M, Hernandez P, et al. CTS position statement: Pharmacotherapy in patients with COPD - An update. Can J Respi Crit Care Sleep Med [Internet]. 2017;1(4):222-41. Available at: https://ctssct.ca/wp-content/uploads/2018/01/Pharmacotherapy-of-COPD-2017. pdf (accessed May 31, 2018).

25. Global Initiative for Chronic Obstructive Lung Disease - Global Initiative for Chronic Obstructive Lung Disease - GOLD. Global Initiative for Chronic Obstructive Lung Disease - GOLD [Internet]. 2018. Available at: http://goldcopd.org/wp-content/uploads/2017/11/GOLD-2018 v6.0-FINAL-revised-20-Nov_WMS.pdf\#\%5B\%7B\%22num\%22\%3A219 \%2C\%22gen\%22\%3A0\%7D\%2C\%7B\%22name\%22\%3A\%22XYZ\% 22\%7D\%2C69\%2C574\%2C0\%5D (accessed June 3, 2018).

26. Clark NM, Zimmerman BJ. A social cognitive view of self-regulated learning about health. Health Educ Res 1990;5(3):371-9. doi: 10.1093/ her/5.3.371.

27. Rosenstock IM, Stretcher VJ, Becker MH. Social learning theory and the Health Belief Model. Health Educ Q 1998;15(2):175-83. doi: 10.1177/109019818801500203.

28. Cancer Prevention Research Centre. Detailed overview: The Transtheoretical Model. Transtheoretical Model [Internet]. The University of Rhode Island; 2018. Available at: https://web.uri.edu/ cprc/detailed-overview/ (accessed October 10, 2018).

29. Alberta Health Services. Alberta Healthy Living Program - Calgary Zone. Service [Internet]. AHS; 2018. Available at: https://www.albertahealthservices.ca/info/service.aspx?id=1005671 (accessed May 31, 2018).

30. Alberta Health Services. AlbertaQuits can help you stop smoking. Albertaquits.ca. [Internet]. 2018. Available at: https://www.albertaquits. $\mathrm{ca} /$ (accessed June 3, 2018).

31. Certification for asthma, respiratory, COPD and tobacco educators. Canadian Network for Respiratory Care| Réseau canadien pour les soins 
respiratoires [Internet]. 2018. Available at: http://cnrchome.net/certifiedoverview.html (accessed June 3, 2018).

32. Lum EY, harpe HM, Nilsson C, et al. Urban and rural differences in the management of asthma amongst primary care physicians in Alberta. Can J Cin Pharmacol 2007;14(3):e275-82.

33. World Health Organization. The mandate for health literacy. 9th Global Conference on Health Promotion . 2018. Available at: http://www.who. int/healthpromotion/conferences/9gchp/health-literacy/en (accessed June 03, 2018).

34. Howcroft M, Walters EH, Wood-Baker R, Walters JA. Action plans with brief patient education for exacerbations in chronic obstructive pulmonary disease. Cochrane Database Syst Rev 2016;12:CD005074. doi: 10.1002/14651858.CD005074.pub4.

35. Pinnock H, Parke HL, Panagioti M, et al. Systematic meta-review of supported self-management for asthma: A healthcare perspective. BMC Med 2017;15:64. doi: 10.1186/s12916-017-0823-7.

36. Alberta Health Services. Online Ambulatory Care Classification System (OACCS) [email communication]. 2018; (accessed 04 June, 2018).

37. Hopman WM, Garvey N, Olajos-Clow J, White-Markham A, Lougheed MD. Outcomes of asthma education: Results of a multisite evaluation. Can Respir J. [Internet] .2004;11(4):291-7. Available at: https://www.hindawi. com/journals/crj/2004/847628/abs/ (accessed October 10, 2018).

38. Peytremann-Bridevaux I, Arditi C, Gex G, Bridevaux PO, Burnand

B. Chronic disease management programmes for adults with asthma. Cochrane Database of Syst Rev 2015;5:CD007988. doi: 10.1002/14651858.CD007988.pub2.

39. Gibson PG, Powell H, Coughlan J, et al. Self-management education and regular practitioner review for adults with asthma. Cochrane Database Syst Rev 2003;1:CD001117.
40. Rees S, Williams A. Promoting and supporting self-management for adults living in the community with physical chronic illness: A systematic review of the effectiveness and meaningfulness of the patient-practitioner encounter. JBI Libr Syst Rev 2009; 7(13):492-582.

41. Kuethe MC, Vaessen-Verberne AA, Elbers RG, Van Aalderen WM. Nurse versus physician-led care for the management of asthma. Cochrane Database Syst Rev 2013;2:CD009296. doi: 10.1002/14651858. CD009296.pub2.

42. Lenferink A, Brusse-Keizer M, van der Valk PD, et al. Self-management interventions including action plans for exacerbations versus usual care in patients with chronic obstructive pulmonary disease. Cochrane Database Syst Rev 2017;8:CD011682. doi: 10.1002/14651858. CD011682.pub2.

43. Murphy LA, Harrington P, Taylor SJ, et al. Clinical-effectiveness of self-management interventions in chronic obstructive pulmonary disease: An overview of reviews. Chron Respir Dis 2017;14(3):276-88.

44. Newham JJ, Presseau J, Heslop-Marshall K, et al. Features of self-management interventions for people with COPD associated with improved health-related quality of life and reduced emergency department visits: A systematic review and meta-analysis. Int J Chron Obstruct Pulmon Dis 2017;12:1705-20. doi: 10.2147/COPD.S133317.

45. Zwerink M, Brusse-Keizer M, van der Valk PD, et al. Self management for patients with chronic obstructive pulmonary disease. Cochrane Database Syst Rev 2014;19(3):CD002990. doi: 10.1002/14651858.CD002990. pub3.

46. Mable AL, Marriott J. Sharing the learning: health transition fund: synthesis series: Primary health care [Internet]. Ottawa, ON: Health Canada; 2002. Available at: http://www.hc-sc.gc.ca/hcs-sss/pubs/acces/2002-htffass-prim/index-eng.php (accessed June 4, 2018). 